\title{
Assembly of Capsules for Irradiation of Silicon Carbide Joint Specimens in the High Flux Isotope Reactor
}

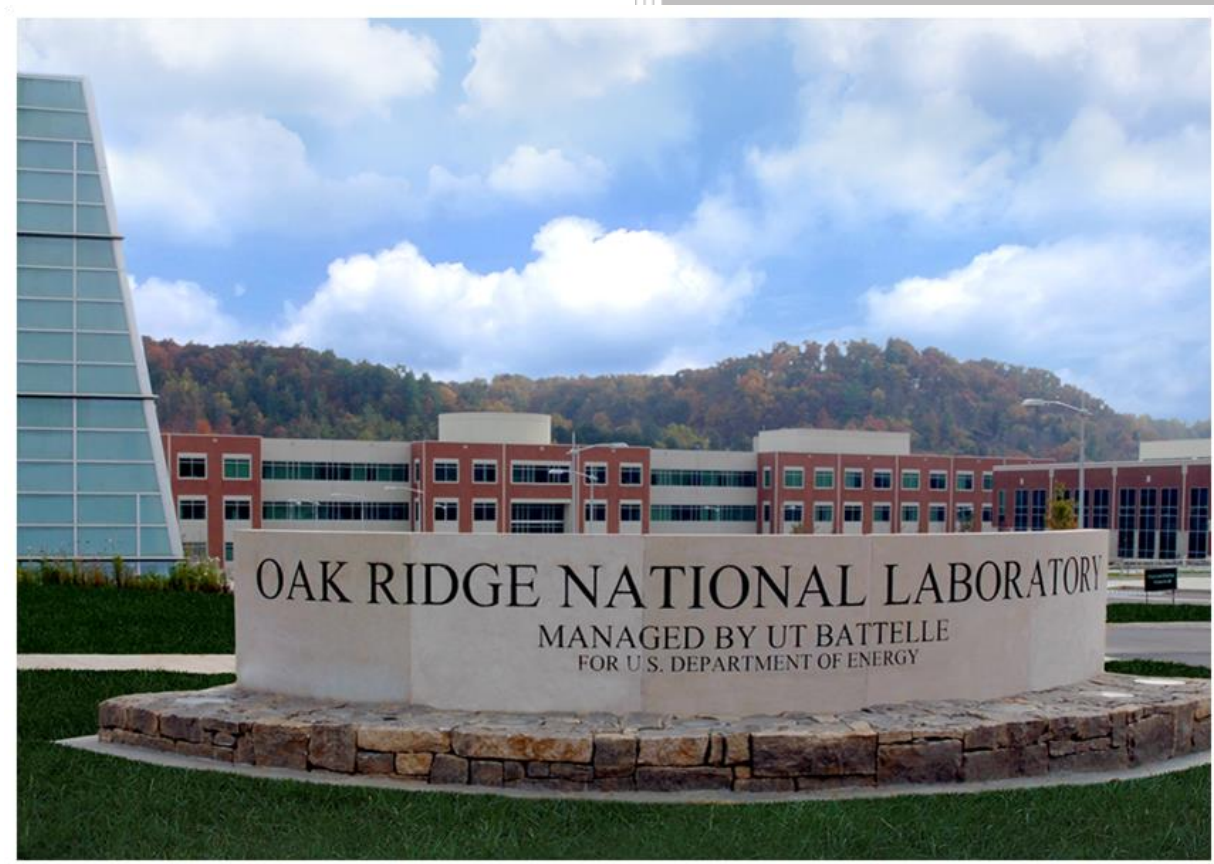

Annabelle G. Le Coq Christian M. Petrie Kory D. Linton Christian P. Deck

November 2019 


\section{DOCUMENT AVAILABILITY}

Reports produced after January 1, 1996, are generally available free via US Department of Energy (DOE) SciTech Connect.

Website www.osti.gov

Reports produced before January 1,1996, may be purchased by members of the public from the following source:

National Technical Information Service

5285 Port Royal Road

Springfield, VA 22161

Telephone 703-605-6000 (1-800-553-6847)

TDD 703-487-4639

Fax 703-605-6900

E-mail info@ntis.gov

Website http://classic.ntis.gov/

Reports are available to DOE employees, DOE contractors, Energy Technology Data Exchange representatives, and International Nuclear Information System representatives from the following source:

Office of Scientific and Technical Information

PO Box 62

Oak Ridge, TN 37831

Telephone 865-576-8401

Fax 865-576-5728

E-mail reports@osti.gov

Website http://www.osti.gov/contact.html

This report was prepared as an account of work sponsored by an agency of the United States Government. Neither the United States Government nor any agency thereof, nor any of their employees, makes any warranty, express or implied, or assumes any legal liability or responsibility for the accuracy, completeness, or usefulness of any information, apparatus, product, or process disclosed, or represents that its use would not infringe privately owned rights. Reference herein to any specific commercial product, process, or service by trade name, trademark, manufacturer, or otherwise, does not necessarily constitute or imply its endorsement, recommendation, or favoring by the United States Government or any agency thereof. The views and opinions of authors expressed herein do not necessarily state or reflect those of the United States Government or any agency thereof. 
Reactor and Nuclear Systems Division

\title{
Assembly of Capsules for Irradiation of Silicon Carbide Joint Specimens in the High Flux Isotope Reactor
}

\author{
Annabelle G. Le Coq \\ Christian M. Petrie \\ Kory D. Linton \\ Christian P. Deck
}

Date Published: November 30, 2019

NSUF Work Package \#: UF-20OR021006

Work Package Manager: Kory Linton

Milestone \#: M3UF-20OR0210062

Prepared by

OAK RIDGE NATIONAL LABORATORY

Oak Ridge, TN 37831-6283

managed by

UT-BATTELLE, LLC

for the

US DEPARTMENT OF ENERGY

under contract DE-AC05-00OR22725 



\section{CONTENTS}

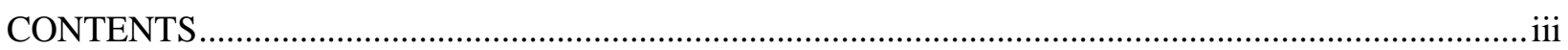

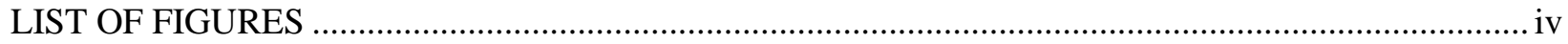

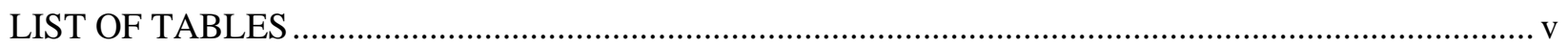

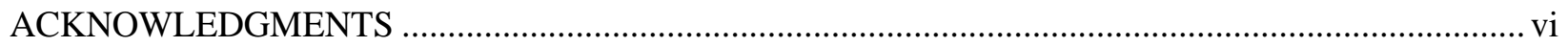

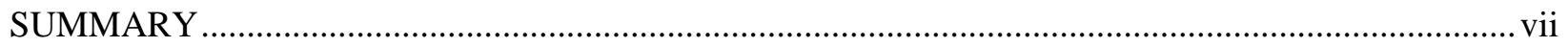

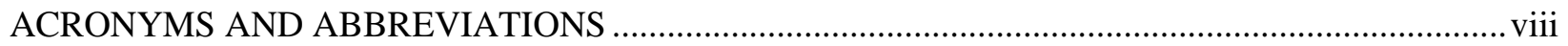

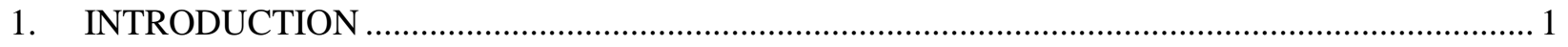

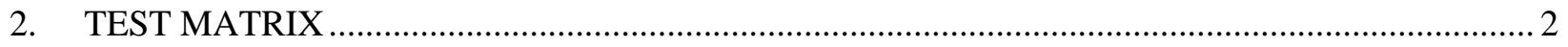

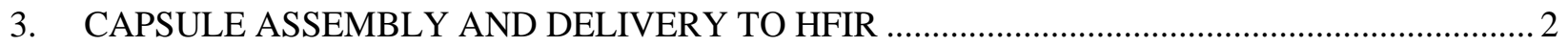

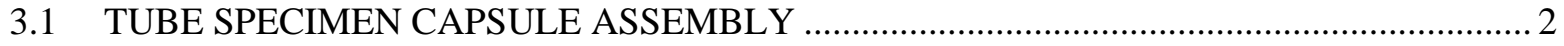

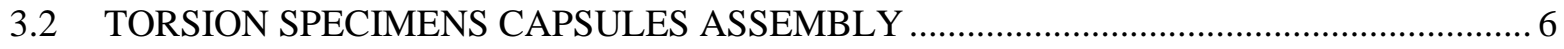

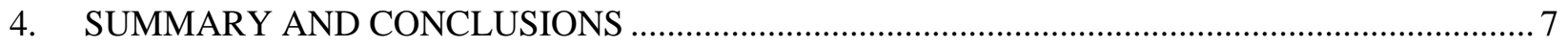

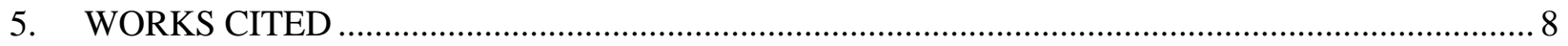

APPENDIX A. FABRICATION REQUEST SHEETS ..................................................................... 


\section{LIST OF FIGURES}

Figure 1. Capsule design concepts for (a) the end plug specimens and (b) the torsion specimens............. 1

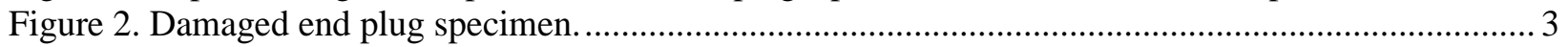

Figure 3. Parts layout for capsules PGA01 through PGA11 .............................................................. 3

Figure 4. Top-down view of (a) the capsule housing and the thermometry inside the tube

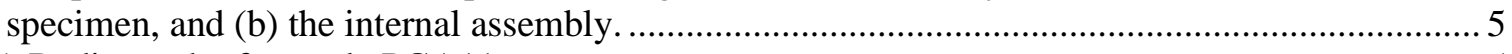

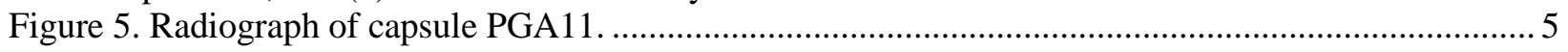

Figure 6. Part layout for (a) capsule TGA01 and (b) capsule TGA02 ................................................ 6

Figure 7. Torsion specimens (a) being setup for insertion in their holder and (b) assembled in the holder and housing. 


\section{LIST OF TABLES}

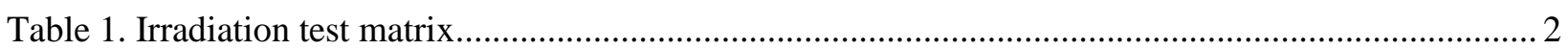




\section{ACKNOWLEDGMENTS}

This research was sponsored by the Nuclear Science User Facilities Program of the US Department of Energy (DOE), Office of Nuclear Energy. Neutron irradiation in the High Flux Isotope Reactor is made possible by the DOE Office of Basic Energy Sciences. The report was authored by UT-Battelle under Contract No. DE-AC05-00OR22725 with DOE. The capsules assembly was performed by David Bryant. 


\section{SUMMARY}

This report provides a summary of the test matrix and assembly of thirteen capsules containing silicon carbide joint specimens, where the goal of this experiment is to investigate the effects of irradiation on such joints. Two capsule designs are used to accommodate two different specimen geometries: a small torsion specimen to measure basic thermomechanical properties, and a cladding-style "end plug" specimen to demonstrate the strength and integrity of such cladding under typical thermal gradients and stresses. The assembled capsules are scheduled for two cycles of irradiation in the flux trap of the High Flux Isotope Reactor with two different target temperatures: 350 and $750^{\circ} \mathrm{C}$. 


\section{ACRONYMS AND ABBREVIATIONS}

$\begin{array}{ll}\text { CVD } & \text { chemical vapor deposition } \\ \text { DOE } & \text { US Department of Energy } \\ \text { GA } & \text { General Atomics } \\ \text { HFIR } & \text { High Flux Isotope Reactor } \\ \text { ORNL } & \text { Oak Ridge National Laboratory } \\ \text { SiC } & \text { silicon carbide } \\ \text { TEP } & \text { transient eutectic phase }\end{array}$




\section{INTRODUCTION}

Silicon carbide ( $\mathrm{SiC}$ ) fiber reinforced $\mathrm{SiC}$ matrix composites (SiC-SiC) are of interest for fuel cladding and structural components in current and advanced nuclear reactor designs because of their strength retention at high temperature, high-temperature steam oxidation resistance, and stability under neutron irradiation [1]. However, to fabricate a complex geometry or to create a hermetic seal, SiC-based reactor components must be joined. While $\mathrm{SiC}$ performance under neutron irradiation is relatively well understood, the performance of the specific joining technologies has received much less attention to date. Any joint technology that will be deployed for in-core applications in current and advanced reactor designs must first demonstrate acceptable irradiation tolerance following irradiation in a materials test reactor.

General Atomics (GA) is currently investigating three joint variations: transient eutectic phase (TEP) SiC-based joints, oxide joints, and high purity SiC-based hybrid joints that use a preceramic polymer with chemical vapor deposition (CVD) SiC. Tube specimens with a single sealed end (i.e., end plug specimens) and torsion specimens have been fabricated using each of the three joint variations. The purpose of this project is to perform experimental irradiation testing of the joint specimens to understand the effects of irradiation with and without realistic temperature gradients, which can drive significant stress [2] [3] [4].

The SiC joint specimens will be inserted into the Oak Ridge National Laboratory (ORNL) High Flux Isotope Reactor (HFIR) using irradiation capsules, or rabbits, which are designed to achieve the desired specimen temperatures during irradiation. The experiment design concepts are detailed in Petrie et al. [5] and are shown in Figure 1. This report presents the irradiation test matrix and summarizes the assembly of thirteen rabbits containing $\mathrm{SiC}$ joint specimens.

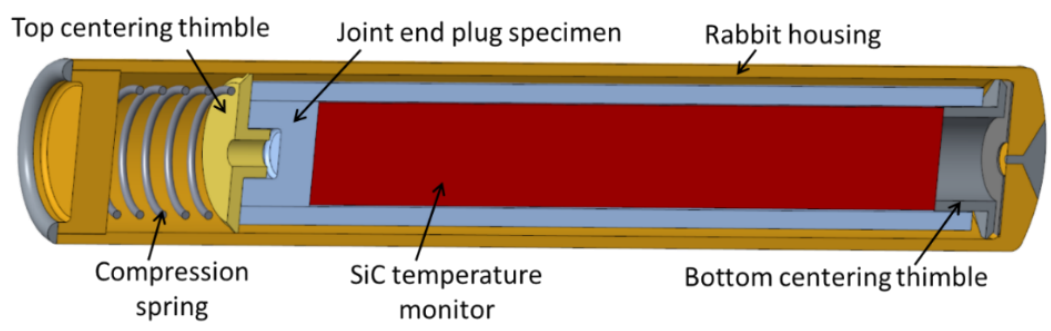

(a)

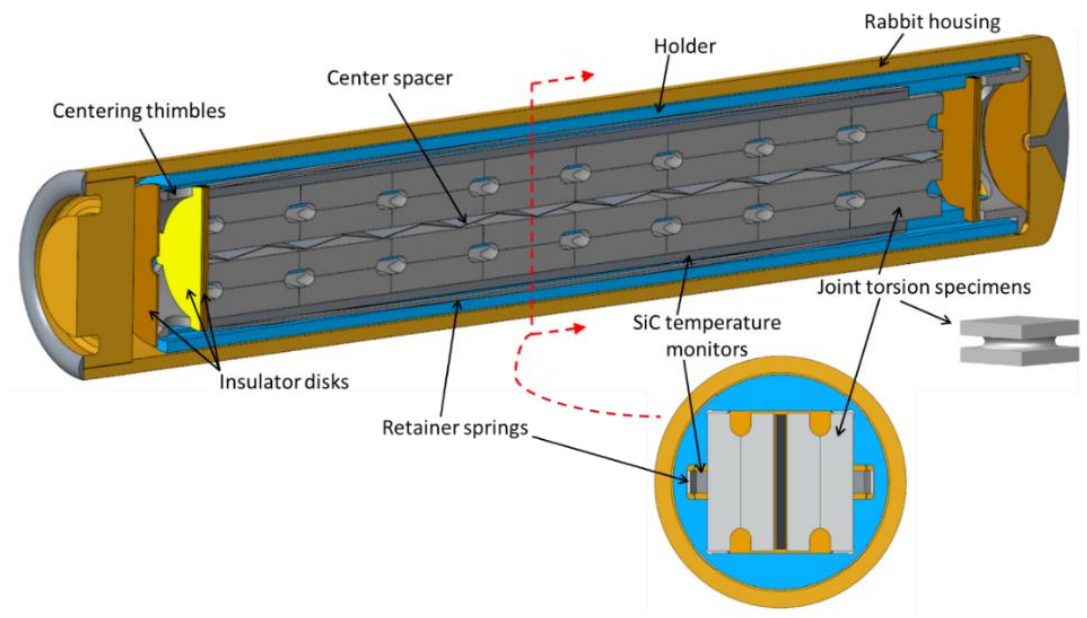

(b)

Figure 1. Capsule design concepts for (a) the end plug specimens and (b) the torsion specimens. 


\section{TEST MATRIX}

Table 1 summarizes the irradiation test matrix, including details on the irradiation conditions, capsule fill gases, and the specimens being inserted in the capsules. Capsule PGA01 through PGA11 accommodate one end plug specimen each, while capsules TGA01 and TGA02 accommodate sixteen torsion specimens each. This test matrix allows the irradiation of each specimen's geometry and each joint type at both target temperatures, $350 \pm 50^{\circ} \mathrm{C}$ and $750 \pm 50^{\circ} \mathrm{C}$. All the capsules will be inserted in axial position 7 of a target rod rabbit holder in the HFIR flux trap for 2 cycles, corresponding to an approximate dose of $2 \mathrm{dpa}$. Position 7 is located at the top of the HFIR core, approximately $19.3 \mathrm{~cm}$ from the core's midplane.

Table 1. Irradiation test matrix

\begin{tabular}{|c|c|c|c|c|}
\hline $\begin{array}{c}\text { Capsule } \\
\text { ID }\end{array}$ & $\begin{array}{c}\text { Temperature } \\
\left({ }^{\circ} \mathrm{C}\right)\end{array}$ & Fill gas & Joint type & Specimen ID \\
\hline PGA01 & 350 & $\mathrm{He}$ & Oxide & GC1 \\
\hline PGA02 & 350 & $\mathrm{He}$ & Oxide & GC2 \\
\hline PGA03 & 750 & $46 \% \mathrm{He}, \mathrm{Ar}$ bal. & Oxide & GC3 \\
\hline PGA04 & 750 & $46 \% \mathrm{He}$, Ar bal. & Oxide & GC7 \\
\hline PGA05 & 350 & $\mathrm{He}$ & Hybrid & HS3 \\
\hline PGA06 & 350 & $\mathrm{He}$ & Hybrid & HS7 \\
\hline PGA07 & 750 & $46 \% \mathrm{He}, \mathrm{Ar}$ bal. & Hybrid & HS8 \\
\hline PGA08 & 750 & $46 \% \mathrm{He}, \mathrm{Ar}$ bal. & TEP & TE2 \\
\hline PGA09 & 750 & $46 \% \mathrm{He}, \mathrm{Ar}$ bal. & Hybrid & HS9 \\
\hline PGA10 & 750 & $46 \% \mathrm{He}, \mathrm{Ar}$ bal. & TEP & TE3 \\
\hline PGA11 & 350 & $\mathrm{He}$ & TEP & TE9 \\
\hline TGA01 & 300 & $\mathrm{He}$ & $\begin{array}{l}\text { TEP } \\
\text { Oxide } \\
\text { Hybrid } \\
\end{array}$ & $\begin{array}{c}\text { 1TE, 3TE, 5TE, 7TE, 9TE } \\
\text { 1GC, 5GC, 10GC, 11GC, 15GC, 18GC } \\
1 \mathrm{HS}, 5 \mathrm{HS}, 9 \mathrm{HS}, 11 \mathrm{HS}, 13 \mathrm{HS}\end{array}$ \\
\hline TGA02 & 800 & $\mathrm{Ar}$ & $\begin{array}{l}\text { TEP } \\
\text { Oxide } \\
\text { Hybrid }\end{array}$ & $\begin{array}{c}\text { 2TE, 4TE, 6TE, 8TE, 10TE, 11TE } \\
\text { 4GC, 7GC, 16GC, 17GC, 20GC } \\
\text { 2HS, 8HS, 14HS, 17HS, 19HS }\end{array}$ \\
\hline
\end{tabular}

\section{CAPSULE ASSEMBLY AND DELIVERY TO HFIR}

\subsection{TUBE SPECIMEN CAPSULE ASSEMBLY}

Eleven capsules, with IDs PGA01 through PGA11 were assembled. A twelfth capsule was initially planned to be built as well, but the twelfth specimen (TE4) was damaged before assembly (see Figure 2). Each capsule contains one end plug specimen, and a single $\mathrm{SiC}$ rod is placed inside the specimen for passive temperature monitoring [6]. The complete parts layout is shown in Figure 3. Figure 4 shows a top-down view of the thermometry fitting inside an end plug specimen, with the specimen assembled inside the capsule housing. Housings were chosen so that the as-built end plug specimen-to-housing gas gap matches the desired $510 \mu \mathrm{m}$ gap as closely as possible. Nine of the eleven capsules have as-built gas gaps within 10 $\mu \mathrm{m}(2 \%)$ of the nominal gap. Capsules PGA02 and PGA03 have slightly larger gaps, as large as $543 \mu \mathrm{m}$ (6.4\% larger than the nominal gap). The radiograph of a capsule in Figure 5 shows the different components assembled in the capsule. 


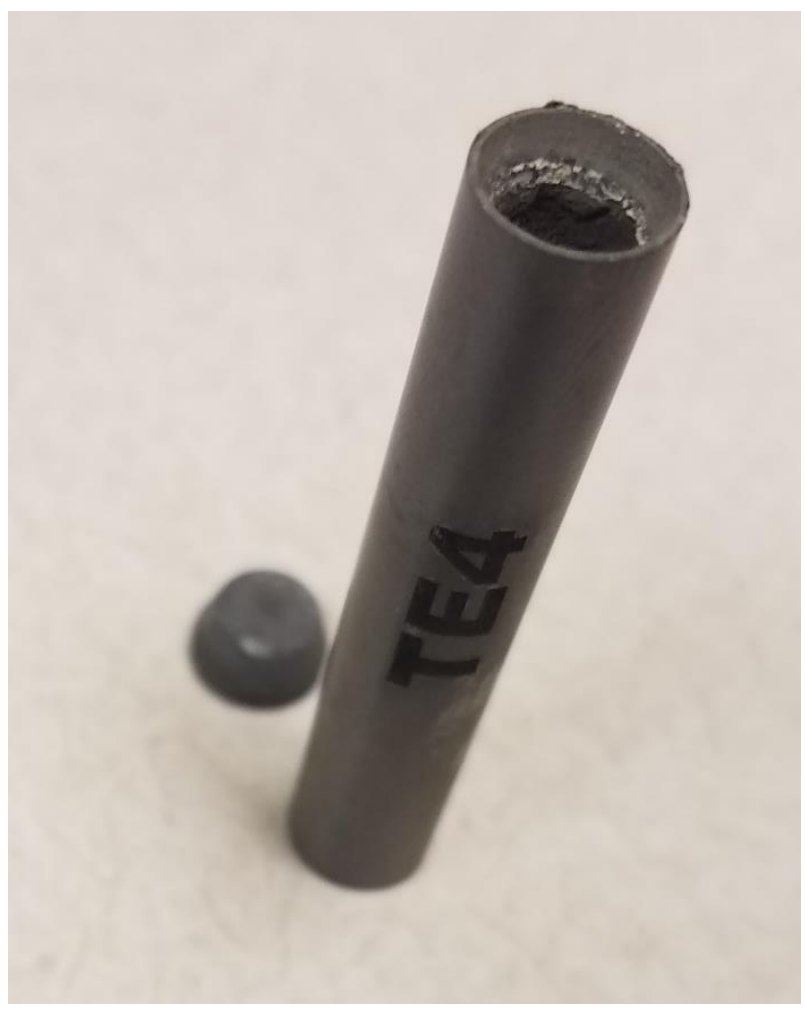

Figure 2. Damaged end plug specimen.

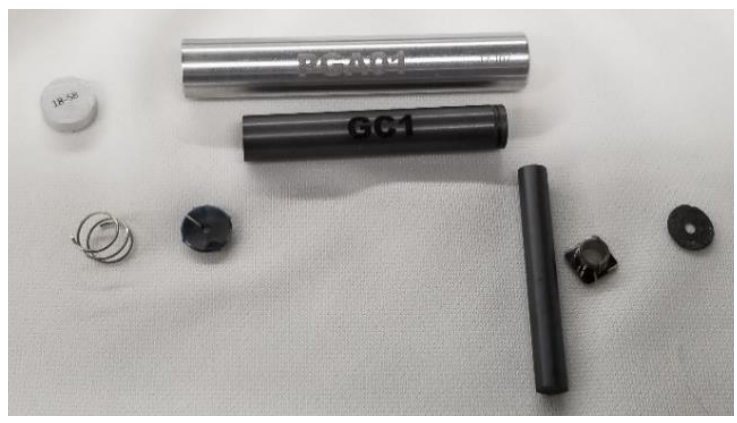

PGA01

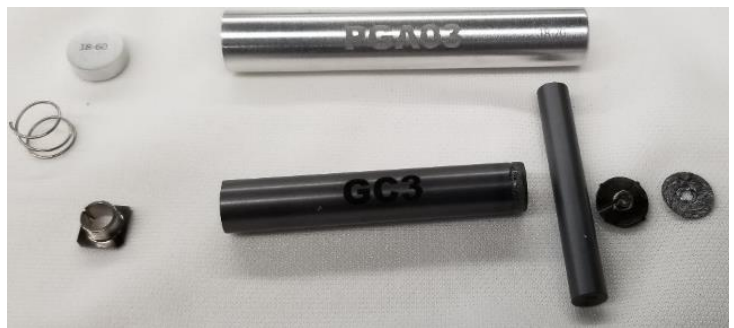

PGA03

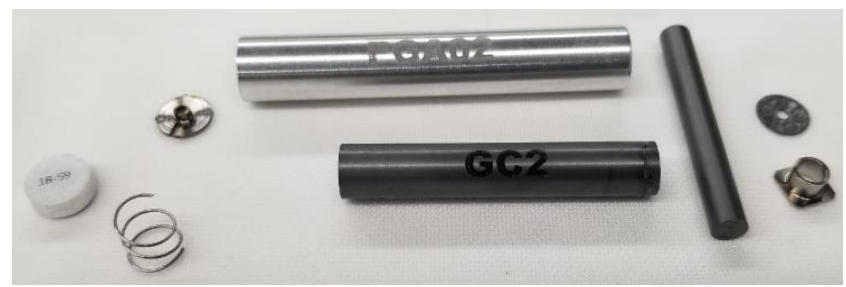

PGA02

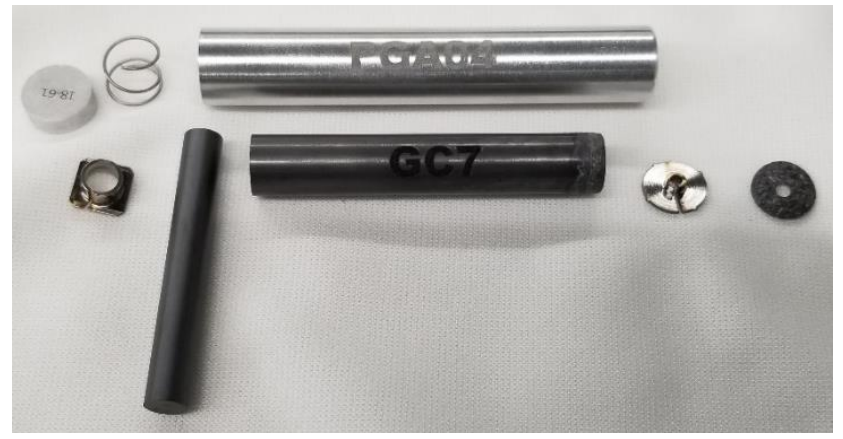

PGA04

Figure 3. Parts layout for capsules PGA01 through PGA11. 


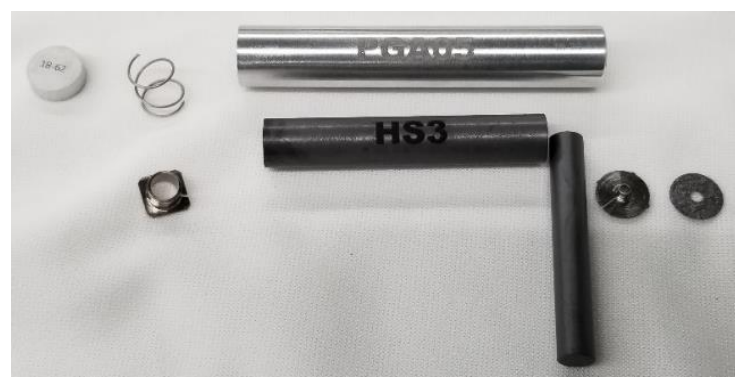

PGA05

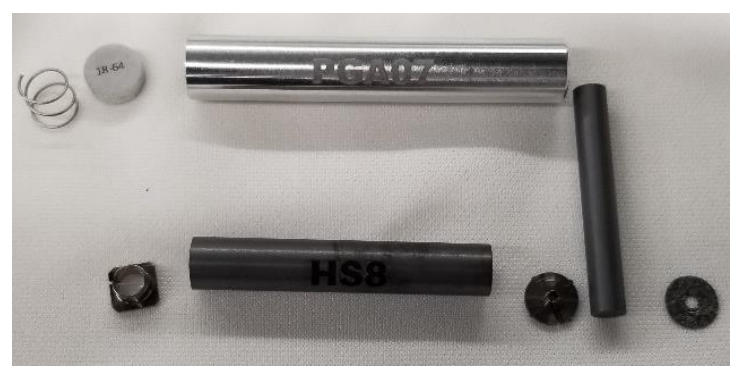

PGA07

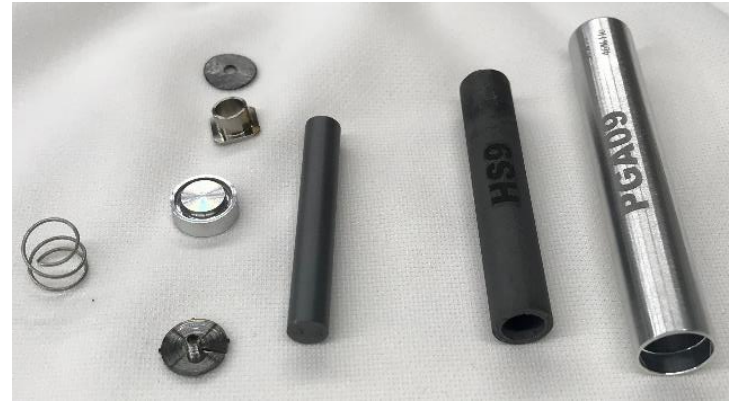

PGA09
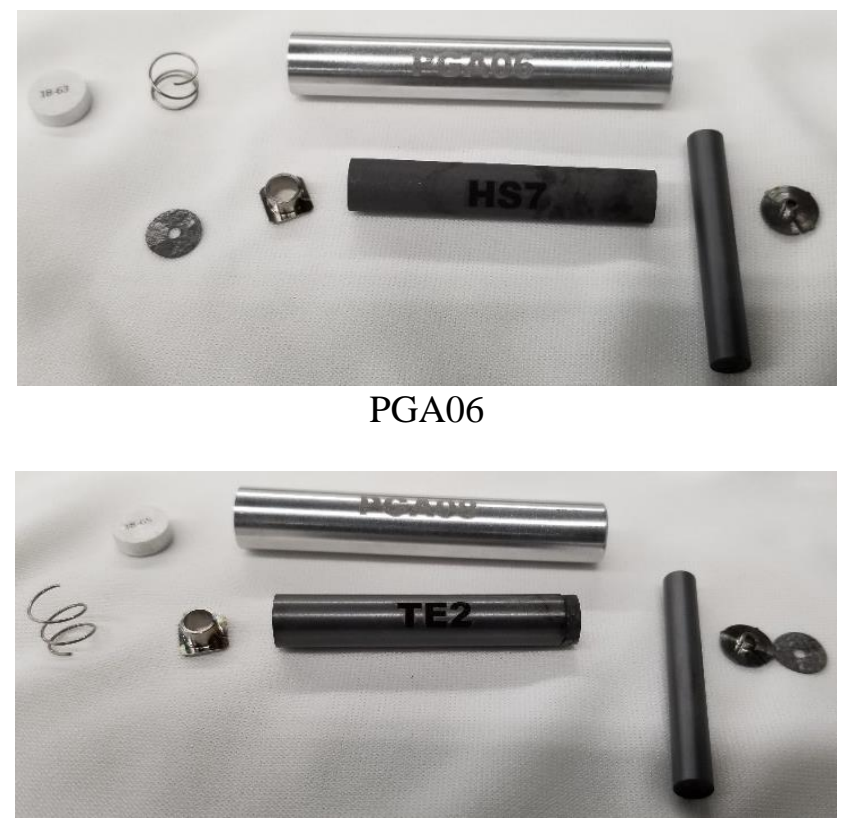

PGA08

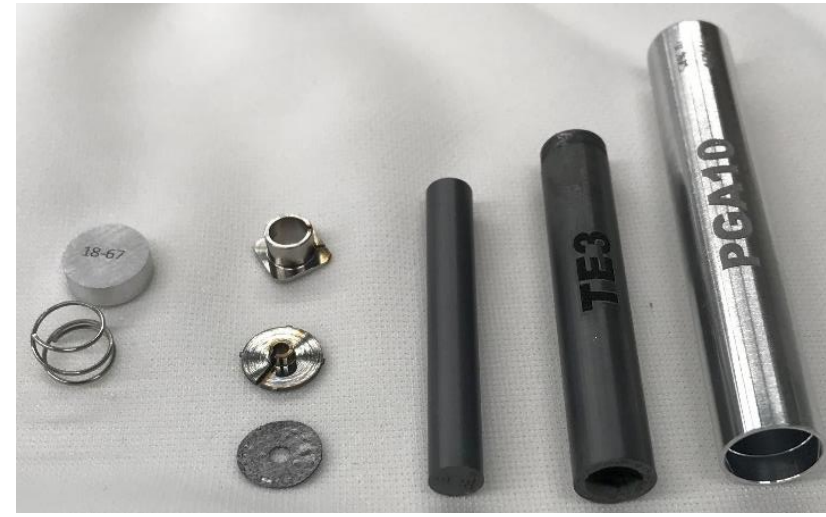

PGA10

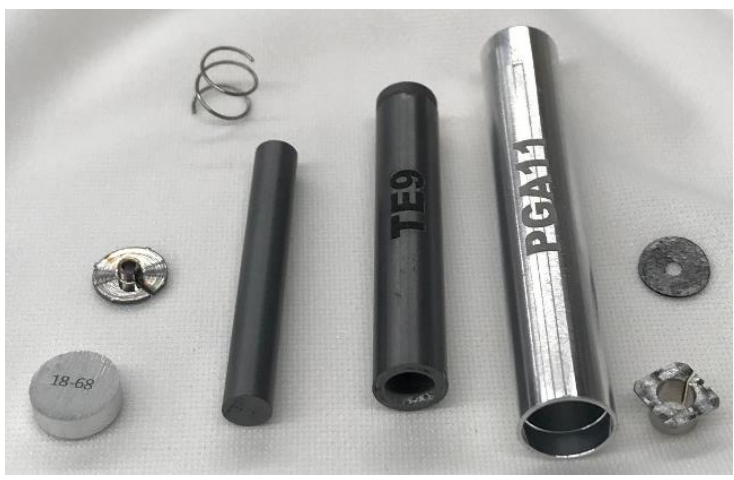

PGA11

Figure 3. Parts layout for capsules PGA01 through PGA11 (continued). 


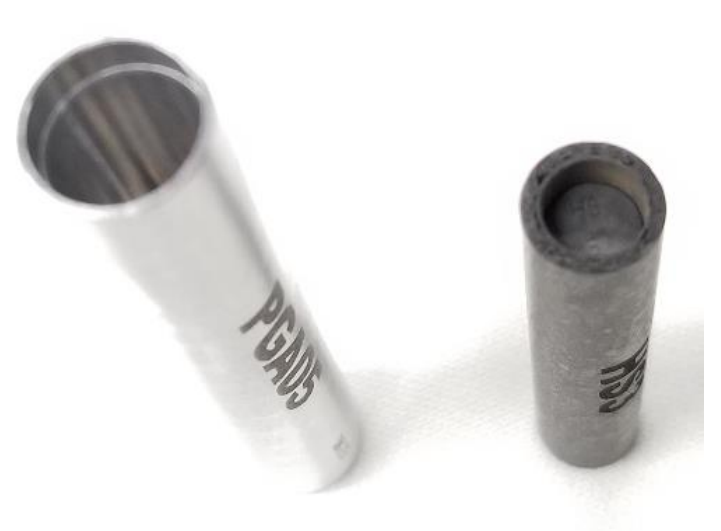

(a)

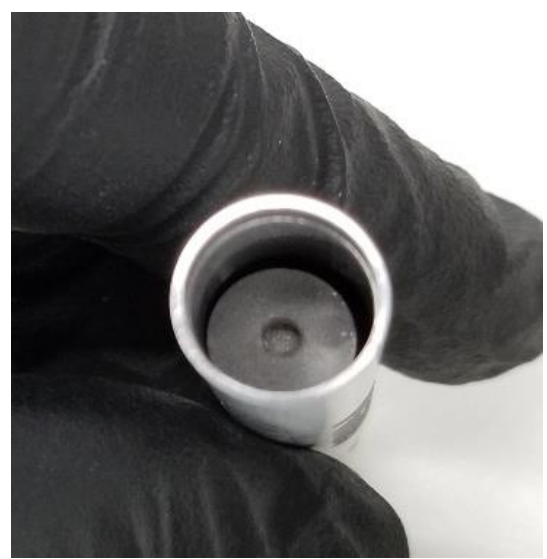

(b)

Figure 4. Top-down view of (a) the capsule housing and the thermometry inside the tube specimen, and (b) the internal assembly.

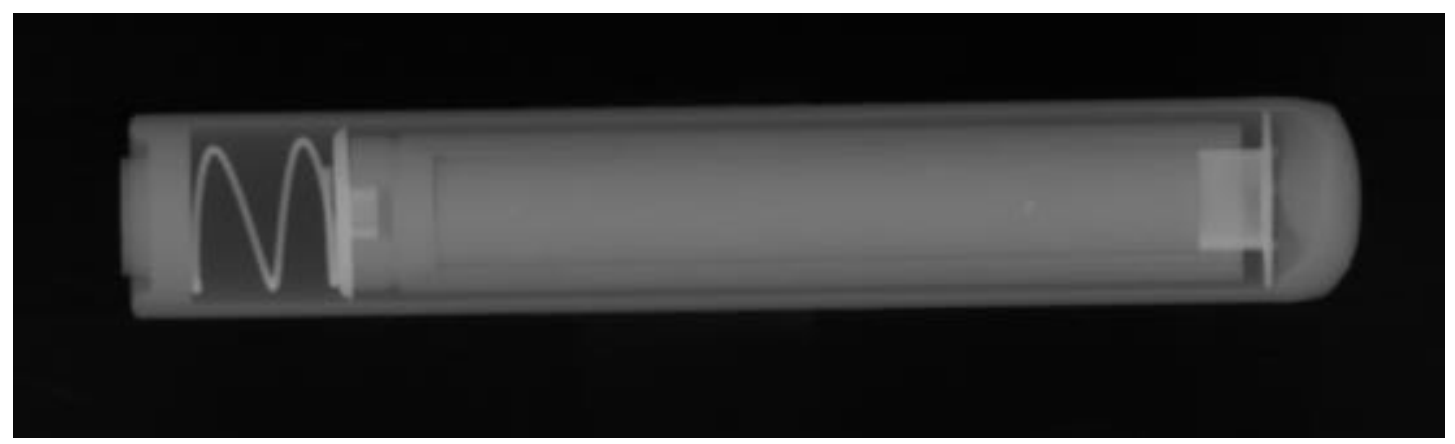

Figure 5. Radiograph of capsule PGA11.

All capsule components were dimensionally inspected and cleaned according to HFIR-approved procedures, drawings, and sketches. After assembly of the internal components, all rabbit housing end caps were welded to the housings using an electron beam weld. The capsules were then placed inside sealed chambers that were evacuated and backfilled three times to ensure a pure environment with the gas mixture specified in Table 1. The chambers were placed inside a glove box, which was also evacuated and backfilled with the same gas mixture. Each rabbit had a small hole in the bottom of the housing that was sealed using a gas tungsten arc welding procedure. All welds passed visual examination. Each capsule was then sent for nondestructive examination, which included a helium leak test, hydrostatic compression at a pressure of 1,035 psi, mass comparisons before and after hydrostatic compression to ensure no water penetrated the capsule housing, and a final post-compression helium leak test. All rabbits passed helium leak testing and hydrostatic compression.

The capsules were delivered to HFIR, along with their fabrication package, which records requirements for design analyses, material certification, assembly procedure, welding, and testing. The signed capsule fabrication request forms are provided in APPENDIX A. 


\subsection{TORSION SPECIMENS CAPSULES ASSEMBLY}

Two capsules containing torsion specimens, TGA01 and TGA02, were assembled. Figure 6 shows the complete part layouts for these two capsules. Each capsule contains sixteen torsion specimens. Figure 7 shows the assembly process, in which all the torsion specimens were lined up before being inserted in their holder and housing. Housings were chosen so that the as-built holder-to-housing gas gap matched the desired gaps within $1 \mu \mathrm{m}(131 \mu \mathrm{m}$ gap for capsule TGA01 and $83 \mu \mathrm{m}$ for capsule TGA02).

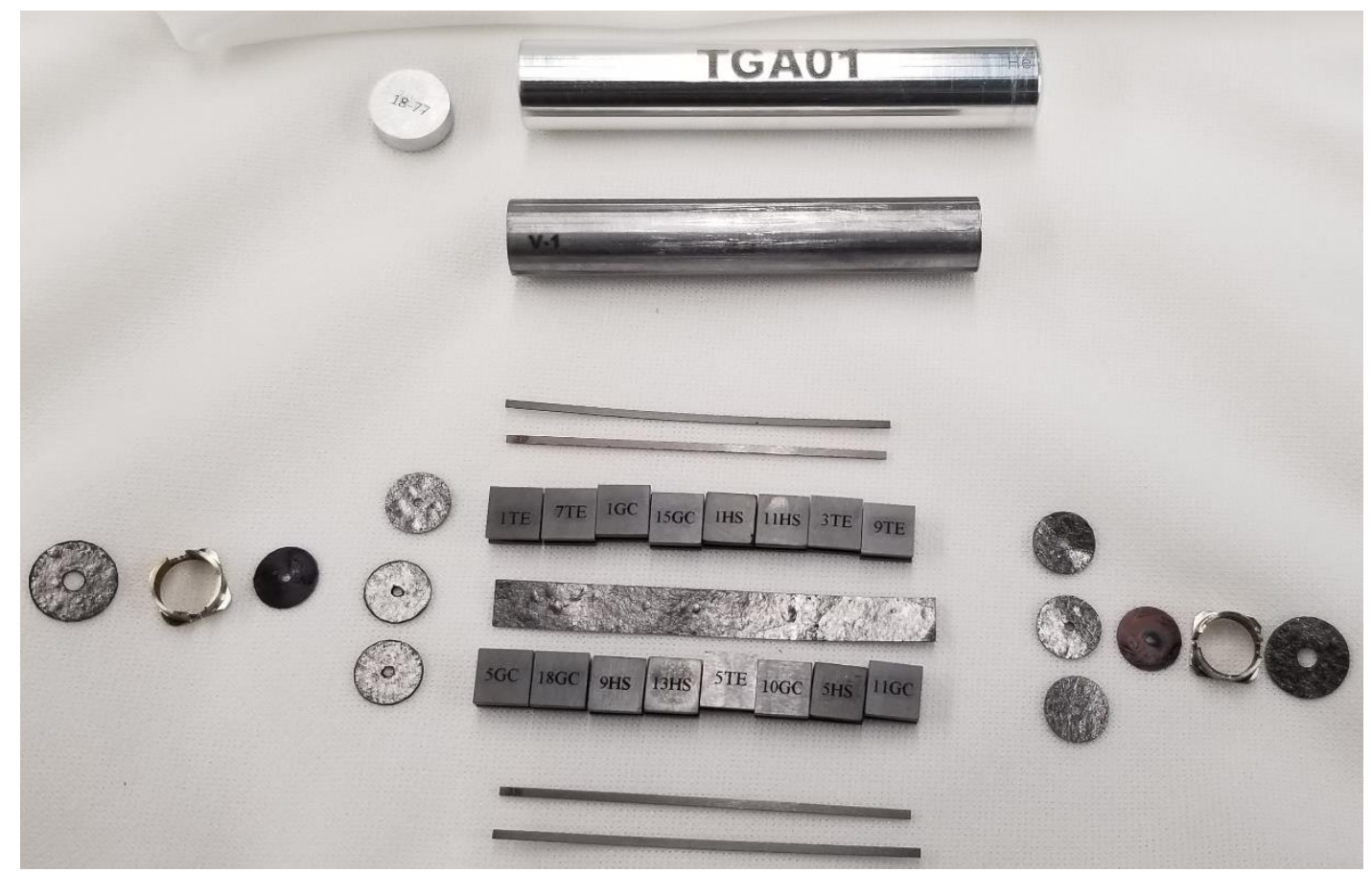

(a)

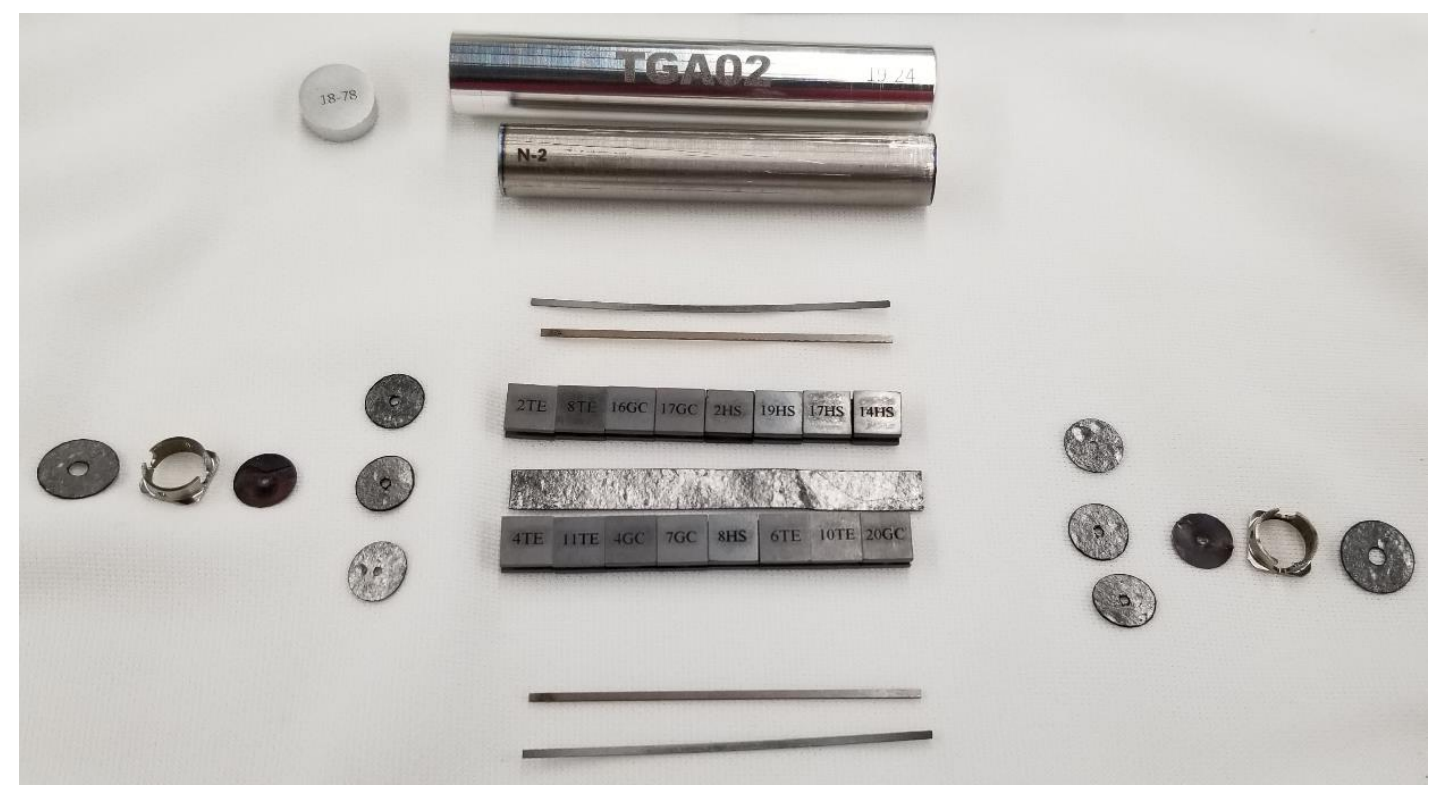

(b)

Figure 6. Part layout for (a) capsule TGA01 and (b) capsule TGA02. 


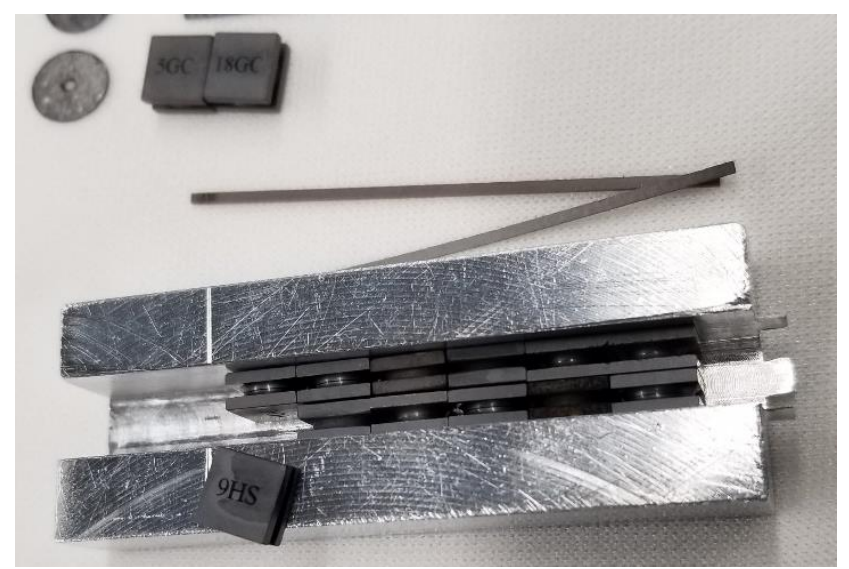

(a)

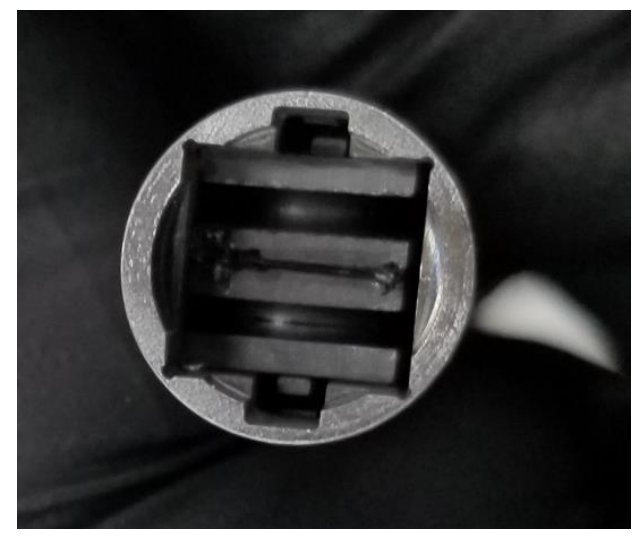

(b)

Figure 7. Torsion specimens (a) being setup for insertion in their holder and (b) assembled in the holder and housing.

Like the end plug specimen capsules, all the components of the torsion specimen capsules were dimensionally inspected and cleaned according to HFIR-approved procedures, drawings, and sketches. The signed capsule fabrication request forms are provided in APPENDIX A.

\section{SUMMARY AND CONCLUSIONS}

This report summarizes the test matrix and assembly of eleven tube specimen rabbit capsules that were successfully delivered to HFIR for insertion during cycle 485 (December 2019). Two torsion specimens rabbit capsules were also assembled. The capsules contain one of two types of specimen geometry (tube or torsion), where each specimen features one of three different joint variations (TEP, oxide, or hybrid). Post-irradiation examination will analyze the joints' mechanical and thermal properties, permeability, and microstructure. Ultimately, the results of this project will aid in understanding the effects of irradiation on $\mathrm{SiC}$ joint performance. 


\section{WORKS CITED}

[1] G. Jacobsen, C. Deck, J. Sheeder, O. Gutierrez, H. Khalifa, and C. Back, "Characterization of SiC-SiC Composties for Accident Tolerant Fuel," Journal of Nuclear Materials, vol. 466, pp. 667-681, 2015.

[2] M. Ben-Belgacem, V. Richet, K.A. Terrani, Y. Katoh, and L.L Snead, "Thermo-Mechanical Analysis of LWR SiC/SiC Composite Cladding," Journal of Nuclear Materials, vol. 447, No. 1-3, pp. 125-142, 2014.

[3] C. M. Petrie, T. Koyanagi, J. L. McDuffee, C. P. Deck, Y. Katoh, and K. A. Terrani, "Experimental Design and Analysis for Irradiation of SiC/SiC Composite Tubes under a Prototypic High Heat Flux," Journal of Nuclear Materials, vol. 491, pp. 94-104, 2017.

[4] G. Singh, K. Terrani, and Y. Katoh, "Thermo-Mechanical Assessment of Full SiC/SiC Composite Cladding for LWR Applications with Sensitivity Analysis," Journal of Nuclear Materials, vol. 499, pp. 126-143, 2018.

[5] C. M. Petrie, A. G. Le Coq, R. C. Gallagher, K. D. Linton, and C. P. Deck, "Design and Thermal Analysis for Irradiation of Silicon Carbide Joint Specimens in the High Flux Isotope Reactor," Oak Ridge National Laboratory, ORNL/TM-2018/940, Oak Ridge, TN, 2018.

[6] K. G. Field et al., "Evaluation of the Continuous Dilatometer Method of Silicon Carbide Thermometry for Passive Irradiation Temperature Determination," Nuclear Instruments and Methods in Physics Research Section B: Beam Interactions with Materials and Atoms, vol. 445, pp. 46-56, 2019. 
APPENDIX A. FABRICATION REQUEST SHEETS 


\section{APPENDIX A. FABRICATION REQUEST SHEETS}

Capsule Number:

Irradiation Conditions Irradiation Location

Design Temperature

First Cycle Goal

Irradiation Time

Fill Gas

Capsule Fabrication

\begin{tabular}{|l|c|c|c|c|}
\cline { 2 - 5 } \multicolumn{1}{c|}{} & Drawing & Rev. & Part & M \\
\hline Housing & X3E020977A634 & $\mathrm{C}$ & 1 & A \\
\hline End Cap & X3EO20977A634 & $\mathrm{C}$ & 2 & A \\
\hline Compression Spring & S17-42-SIC_ENDPLUG & 0 & 2 & 3 \\
\hline Insulator Disk & S17-42-SIC_ENDPLUG & 0 & 3 & \\
\hline End Plug Specimen & S17-43-SIC_ENDPLUG & 1 & 1 & \\
\hline Bottom Centering Thimble & S17-43-SIC_ENDPLUG & 1 & 2 & Ti-6A \\
\hline Thermometry & S17-43-SIC_ENDPLUG & 1 & 3 & \\
\hline Top Centering Thimble & S17-43-SIC_ENDPLUG & 1 & 4 & Ti \\
\hline
\end{tabular}

Assembly

Assembly Drawing

Welding \& Cleaning

Fill Gas
PGA01

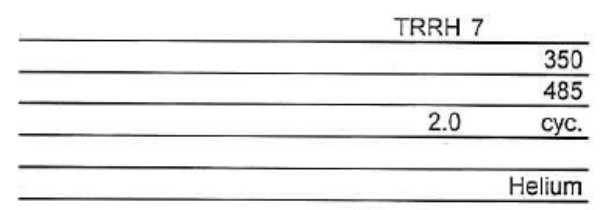

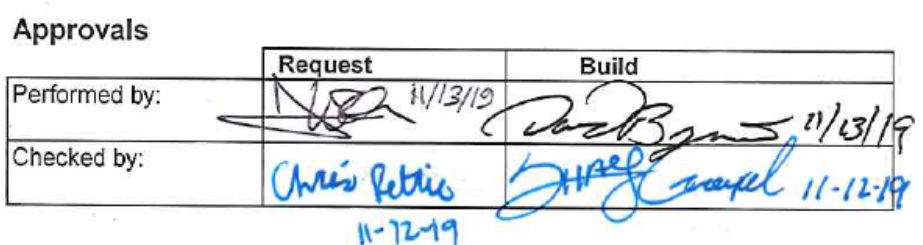

\begin{tabular}{|c|c|c|c|c|c|c|}
\hline laterial & Count & Comment & MAT IR & FAB IR & ID & Mass (g) \\
\hline 6061 & 1 & & 20713 & 20713 & $17-107$ & 4.3016 \\
\hline 4047 & 1 & & 20823 & 20850 & $18-58$ & 0.6155 \\
\hline 304 SS & 1 & & 20659 & 21006 & 01 & 0.1088 \\
\hline Grafoil & 1 & & 19812 & 19812 & 1 total & 0.0088 \\
\hline $\mathrm{sic}$ & 1 & GC1 & 20992 & 20992 & CA TUBE 1 & 4.4851 \\
\hline $\mathrm{i}-6 \mathrm{Al} / 4 \mathrm{~V}$ & 1 & $\varnothing 5.48 \pm 0.03$ & 20950 & 20950 & 1 total & 0.2196 \\
\hline Sic & 1 & & 19759 & 20948 & CA 1 & 3.0635 \\
\hline $\mathrm{i}-6 \mathrm{Al} / 4 \mathrm{~V}$ & 1 & & 20950 & 20950 & $18-01$ & 0.2257 \\
\hline & & total mass & 13.0286 \\
\hline & & & & & specimen mass & 4.4851 \\
\hline
\end{tabular}

specimen mass 


\section{Capsule Number:}

Irradiation Conditions

Irradiation Location

Design Temperature

First Cycle Goal

Irradiation Time

Fill Gas

PGA02

TRRH 7

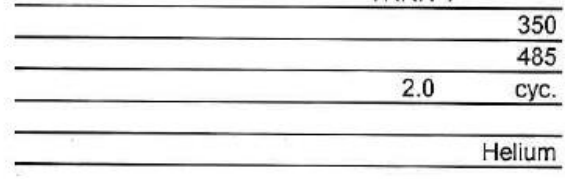

\section{Approvals}

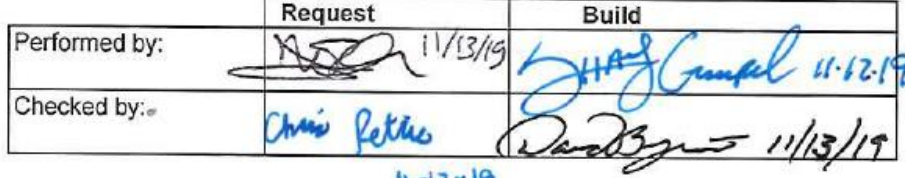

\section{Capsule Fabrication}

\begin{tabular}{|c|c|c|c|c|c|c|c|c|c|c|}
\hline & Drawing & Rev. & Part & Material & Count & Comment & MAT IR & FAB IR & ID & Mass (g) \\
\hline Housing & X3E020977A634 & $\mathrm{C}$ & 1 & Al 6061 & 1 & & 20713 & 20713 & $17-135$ & 4.3083 \\
\hline End Cap & X3E020977A634 & $\mathrm{C}$ & 2 & Al 4047 & 1 & & 20823 & 20850 & $18-59$ & 0.6153 \\
\hline Compression Spring & S17-42-SIC_ENDPLUG & 0 & 2 & 304 SS & 1 & & 20659 & 21006 & 02 & 0.1066 \\
\hline Insulator Disk & S17-42-SIC_ENDPLUG & 0 & 3 & Grafoil & 1 & & 19812 & 19812 & 1 total & 0.0087 \\
\hline End Plug Specimen & S17-43-SIC_ENDPLUG & 1 & 1 & $\mathrm{SiC}$ & 1 & $\mathrm{GC2}$ & 20992 & 20992 & CA TUBE 2 & 4.2169 \\
\hline Bottom Centering Thimble & S17-43-SIC_ENDPLUG & 1 & 2 & Ti-6Al4V & 1 & $\varnothing 5.58 \pm 0.03$ & 20950 & 20950 & 1 total & 0.2144 \\
\hline Thermometry & S17-43-SIC_ENDPLUG & 1 & 3 & $\mathrm{SiC}$ & 1 & & 19759 & 20948 & CA 2 & 3.0692 \\
\hline Top Centering Thimble & S17-43-SIC_ENDPLUG & 1 & 4 & Ti-6Al4V & 1 & & 20950 & 20950 & $18-02$ & 0.2253 \\
\hline & & & & & & & & & total mass & 12.7647 \\
\hline
\end{tabular}

\section{Assembly}

\begin{tabular}{|l|c|c|c|}
\cline { 2 - 4 } \multicolumn{1}{c|}{} & \multicolumn{1}{c|}{ Drawing } & Rev. & \multicolumn{1}{c|}{ Comment } \\
\hline Assembly Drawing & S17-42-SIC_ENDPLUG & 0 & \\
\hline Welding \& Cleaning & X3E020977A633 & 2 & \\
\hline Fill Gas & \multicolumn{3}{|c|}{ Helium } \\
\hline
\end{tabular}


Capsule Number:

Irradiation Conditions

Irradiation Location

Design Temperature

First Cycle Goal

Irradiation Time

Fill Gas

Capsule Fabrication

\begin{tabular}{|c|c|c|c|c|}
\hline & Drawing & Rev. & Part & Material \\
\hline Housing & X3E020977A634 & C & 1 & Al 6061 \\
\hline End Cap & X3E020977A634 & C & 2 & Al 4047 \\
\hline Compression Spring & S17-42-SIC_ENDPLUG & 0 & 2 & 304 SS \\
\hline Insulator Disk & S17-42-SIC_ENDPLUG & 0 & 3 & Grafoil \\
\hline End Plug Specimen & S17-43-SIC_ENDPLUG & 1 & 1 & $\mathrm{SiC}$ \\
\hline Bottom Centering Thimble & S17-43-SIC_ENDPLUG & 1 & 2 & Ti-6AI4V \\
\hline Thermometry & S17-43-SIC_ENDPLUG & 1 & 3 & $\mathrm{SiC}$ \\
\hline Top Centering Thimble & S17-43-SIC_ENDPLUG & 1 & 4 & Ti-6Al4V \\
\hline \multirow[t]{2}{*}{ Assembly } & & & & \\
\hline & Drawing & Rev. & & Comment \\
\hline Assembly Drawing & S17-42-SIC_ENDPLUG & 0 & & \\
\hline Welding \& Cleaning & X3E020977A633 & 2 & & \\
\hline Fill Gas & & & \multicolumn{2}{|c|}{$46 \% \mathrm{He}$, Ar bal. } \\
\hline
\end{tabular}

$46 \% \mathrm{He}$, Ar bal.

TRRH 7

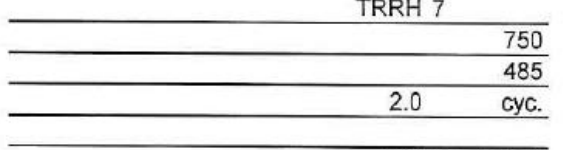

Approvals

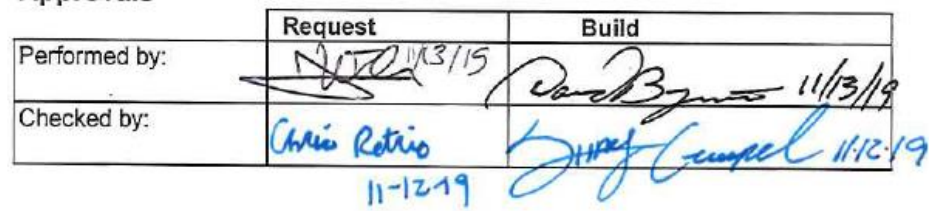
$11-121^{\circ}$ 0 r

\begin{tabular}{|c|c|c|c|}
\hline R & FAB IR & ID & Mass $(\mathbf{g})$ \\
\hline 8 & 20839 & $18-20$ & 4.3098 \\
3 & 20850 & $18-60$ & 0.6120 \\
\hline 9 & 21006 & 03 & 0.1011 \\
2 & 19812 & 1 total & 0.0087 \\
2 & 20992 & CA TUBE 3 & 4.1171 \\
0 & 20950 & 1 total & 0.2187 \\
9 & 20948 & CA 3 & 3.3952 \\
\hline 0 & 20950 & $18-03$ & 0.2242 \\
\cline { 2 - 4 } & total mass & 12.9868 \\
\cline { 2 - 4 } & specimen mass & 4.1171 \\
\cline { 2 - 4 } & &
\end{tabular}


Irradiation Conditions

Irradiation Location

Design Temperature

First Cycle Goal

Irradiation Time

Fill Gas

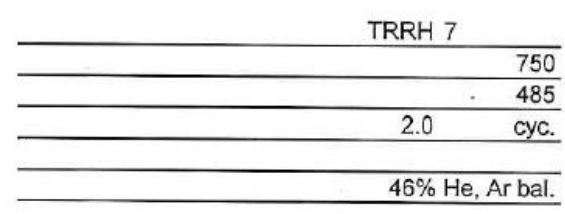

\section{Approvals}

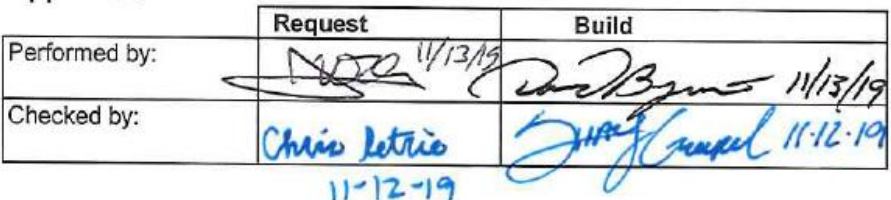

\section{Capsule Fabrication}

\begin{tabular}{|c|c|c|c|c|c|c|c|c|c|c|}
\hline & Drawing & Rev. & Part & Material & Count & Comment & MAT IR & FAB IR & ID & Mass (g) \\
\hline Housing & X3E020977A634 & $\mathrm{C}$ & 1 & Al 6061 & 1 & & 20838 & 20839 & $18-21$ & 4.2986 \\
\hline End Cap & X3E020977A634 & $c$ & 2 & Al 4047 & 1 & & 20823 & 20850 & $18-61$ & 0.6149 \\
\hline Compression Spring & S17-42-SIC_ENDPLUG & 0 & 2 & 304 SS & 1 & & 20659 & 21006 & 04 & 0.0994 \\
\hline Insulator Disk & S17-42-SIC_ENDPLUG & 0 & 3 & Grafoil & 1 & & 19812 & 19812 & 1 total & 0.0084 \\
\hline End Plug Specimen & S17-43-SIC_ENDPLUG & 1 & 1 & $\mathrm{SiC}$ & 1 & GC7 & 20992 & 20992 & CA TUBE 7 & 4.3983 \\
\hline Bottom Centering Thimble & S17-43-SIC_ENDPLUG & 1 & 2 & Ti-6AI4V & 1 & $\varnothing 5.48 \pm 0.03$ & 20950 & 20950 & 1 total & 0.2066 \\
\hline Thermometry & S17-43-SIC_ENDPLUG & 1 & 3 & $\mathrm{SiC}$ & 1 & & 19759 & 20948 & CA 7 & 3.0774 \\
\hline Top Centering Thimble & S17-43-SIC_ENDPLUG & 1 & 4 & Ti-6AI4V & 1 & & 20950 & 20950 & $18-04$ & 0.2249 \\
\hline & & & & & & & & & total mass & 12.9285 \\
\hline
\end{tabular}

Assembly

\begin{tabular}{|l|c|c|c|}
\cline { 2 - 4 } \multicolumn{1}{c|}{} & \multicolumn{1}{c|}{ Drawing } & Rev. & Comment \\
\hline Assembly Drawing & S17-42-SIC_ENDPLUG & 0 & \\
\hline Welding \& Cleaning & X3E020977A633 & 2 & \\
\hline Fill Gas & \multicolumn{2}{|c|}{$46 \% \mathrm{He}$, Ar bal. } \\
\hline
\end{tabular}




\section{Capsule Number:}

Irradiation Conditions

Irradiation Location

Design Temperature

First Cycle Goa

Irradiation Time

Fill Gas
PGA05

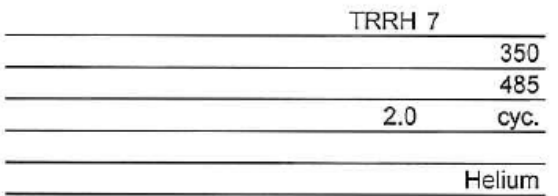

Approvals

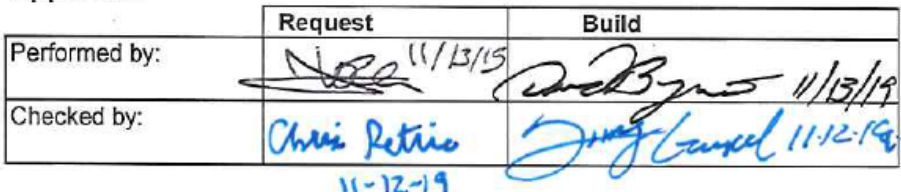

Capsule Fabrication

\begin{tabular}{|c|c|c|c|c|c|c|c|c|c|c|}
\hline & Drawing & Rev. & Part & Material & Count & Comment & MAT IR & FAB IR & ID & Mass (g) \\
\hline Housing & X3E020977A634 & $c$ & 1 & Al 6061 & 1 & & 20838 & 20839 & $18-23$ & 4.2962 \\
\hline End Cap & X3E020977A634 & $\mathrm{c}$ & 2 & Al 4047 & 1 & & 20823 & 20850 & $18-62$ & 0.6157 \\
\hline Compression Spring & S17-42-SIC_ENDPLUG & 0 & 2 & 304 SS & 1 & & 20659 & 21006 & 05 & 0.1023 \\
\hline Insulator Disk & S17-42-SIC_ENDPLUG & 0 & 3 & Grafoil & 1 & & 19812 & 19812 & 1 total & 0.0078 \\
\hline End Plug Specimen & S17-43-SIC_ENDPLUG & 1 & 1 & $\mathrm{SiC}$ & 1 & HS3 & 20992 & 20992 & HSiC TUBE 3 & 4.0204 \\
\hline Bottom Centering Thimble & S17-43-SIC_ENDPLUG & 1 & 2 & Ti-6Al4V & 1 & $\varnothing 5.76 \pm 0.03$ & 20950 & 20950 & 1 total & 0.2138 \\
\hline Thermometry & S17-43-SIC_ENDPLUG & 1 & 3 & $\mathrm{SiC}$ & 1 & & 19759 & 20948 & HS3 & 3.5322 \\
\hline Top Centering Thimble & S17-43-SIC_ENDPLUG & 1 & 4 & Ti-6Al4V & 1 & & 20950 & 20950 & $18-05$ & 0.2237 \\
\hline & & & & & & & & & total mass & 13.0121 \\
\hline
\end{tabular}

Assembly

\begin{tabular}{|l|c|c|c|}
\cline { 2 - 4 } \multicolumn{1}{c|}{} & \multicolumn{1}{c|}{ Drawing } & Rev. & Comment \\
\hline Assembly Drawing & S17-42-SIC_ENDPLUG & 0 & \\
\hline Welding \& Cleaning & X3E020977A633 & 2 & \\
\hline Fill Gas & \multicolumn{3}{|c|}{ Helium } \\
\hline
\end{tabular}




\section{Capsule Number:}

Irradiation Conditions

Irradiation Location

Design Temperature

First Cycle Goa

Irradiation Time

Fill Gas

Capsule Fabrication

\section{Capsule Fabrication}

\begin{tabular}{|l|}
\hline Housing \\
\hline End Cap \\
\hline Compression Spring \\
\hline
\end{tabular}

Compression Spring

nsulator Disk

End Plug Specimen

Bottom Centering Thimble

Thermometry

Top Centering Thimble

$+2$

(n)

\begin{tabular}{l|l} 
& S17-43-SIC_ENDPLUG \\
\hline & S17-43-SIC_ENDPLUG
\end{tabular}

\begin{tabular}{c|c|c|c} 
Drawing & Rev. & Part & \\
X3E020977A634 & $C$ & 1 & \\
X3E020977A634 & $C$ & 2 & \\
S17-42-SIC_ENDPLUG & 0 & 2 & \\
\hline S17-42-SIC_ENDPLUG & 0 & 3 & \\
\hline S17-43-SIC_ENDPLUG & 1 & 1 & \\
\hline S17-43-SIC_ENDPLUG & 1 & 2 & \\
\hline S17-43-SIC_ENDPLUG & 1 & 3 & \\
\hline S17-43-SIC_ENDPLUG & 1 & 4 &
\end{tabular}

\begin{tabular}{|r|r|}
\hline & Mate \\
\hline Al 60 \\
\hline Al 4047 \\
\hline & 304 \\
\hline & Gra \\
\hline & Si \\
\hline & Ti 6 S \\
\hline
\end{tabular}

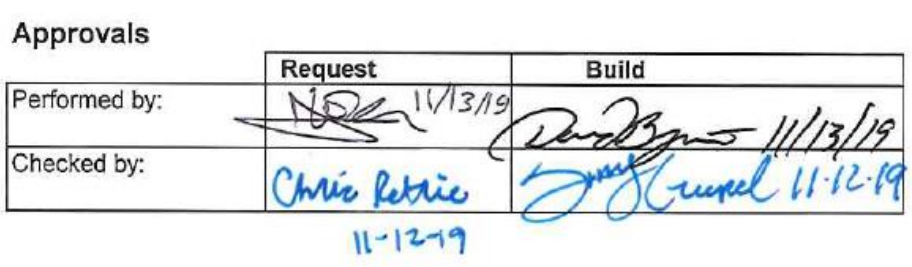

Assembly

\begin{tabular}{|c|c|c|c|}
\hline & Drawing & Rev. & Commen \\
\hline Assembly Drawing & S17-42-SIC_ENDPLUG & 0 & \\
\hline Welding \& Cleaning & X3E020977A633 & 2 & \\
\hline Fill Gas & & & Helium \\
\hline
\end{tabular}




\section{Capsule Number:}

Irradiation Conditions Irradiation Location

Design Temperature

First Cycle Goal

Irradiation Time

Fill Gas

\section{Capsule Fabrication}

\section{Capsule Fabrication}

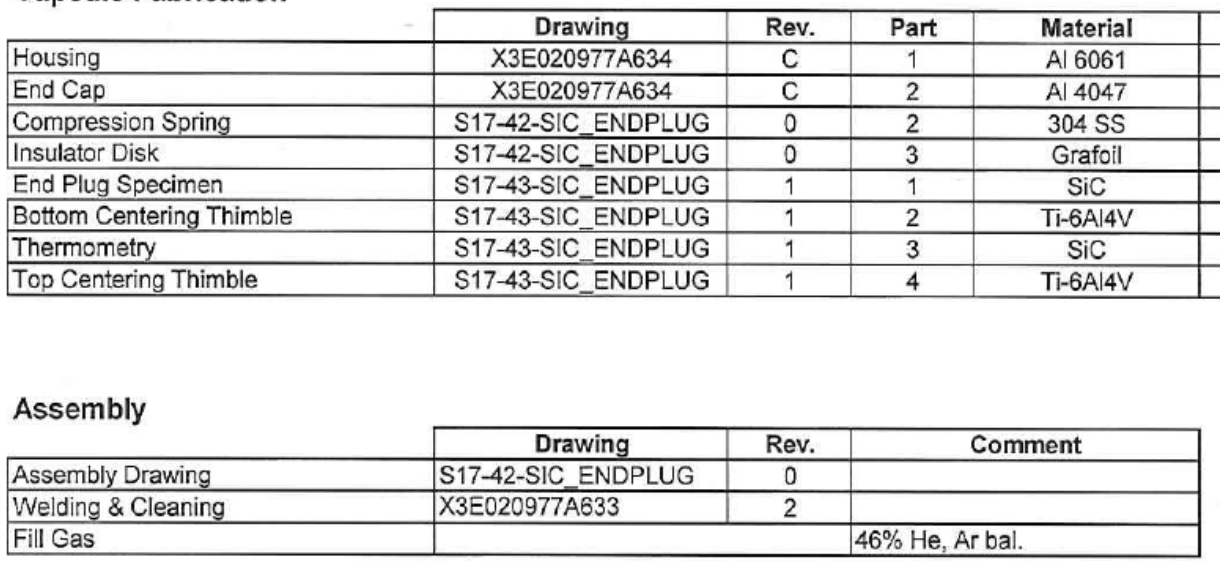

PGA07

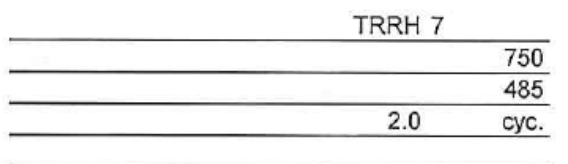

$46 \% \mathrm{He}, \mathrm{Ar}$ bal.

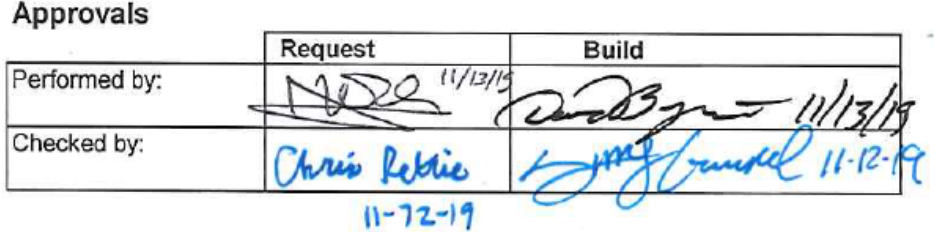


Capsule Number

Irradiation Conditions

Irradiation Location

Design Temperature

First Cycle Goal

Irradiation Time

Fill Gas

$\mathrm{RRH} 7$

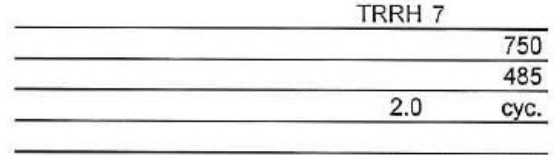

$46 \% \mathrm{He}$, Ar bal.
Approvals

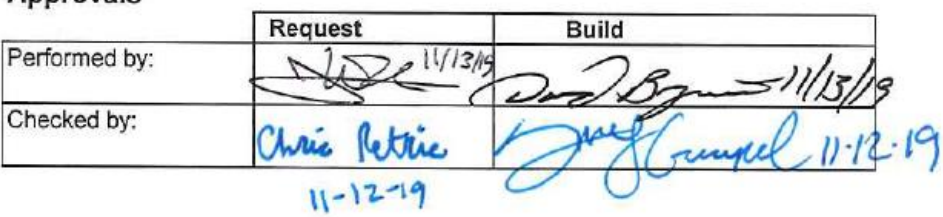

Capsule Fabrication

\begin{tabular}{|c|c|c|c|c|c|c|c|c|c|c|}
\hline & Drawing & Rev. & Part & Material & Count & Comment & MATIR & FAB IR & ID & Mass (g) \\
\hline Housing & X3E020977A634 & C & 1 & Al 6061 & 1 & & 20838 & 20839 & $18-71$ & 4.3454 \\
\hline End Cap & X3E020977A634 & C & 2 & Al 4047 & 1 & & 20823 & 20850 & $18-65$ & 0.6158 \\
\hline Compression Spring & S17-42-SIC_ENDPLUG & 0 & 2 & 304 SS & 1 & & 20659 & 21006 & 08 & 0.0967 \\
\hline Insulator Disk & S17-42-SIC_ENDPLUG & 0 & 3 & Grafoil & 1 & & 19812 & 19812 & 1 total & 0.0087 \\
\hline End Plug Specimen & S17-43-SIC_ENDPLUG & 1 & 1 & $\mathrm{SiC}$ & 1 & TE2 & 20992 & 20992 & TEP TUBE 2 & 4.2049 \\
\hline Bottom Centering Thimble & S17-43-SIC_ENDPLUG & 1 & 2 & Ti-6Al4V & 1 & $\varnothing 5.58 \pm 0.03$ & 20950 & 20950 & 1 total & 0.2156 \\
\hline Thermometry & S17-43-SIC_ENDPLUG & 1 & 3 & $\mathrm{SiC}$ & 1 & & 19759 & 20948 & TEP2 & 3.2345 \\
\hline Top Centering Thimble & S17-43-SIC_ENDPLUG & 1 & 4 & Ti-6AI4V & 1 & & 20950 & 20950 & $18-08$ & 0.2259 \\
\hline & & & & & & & & & $\begin{array}{l}\text { total mass } \\
\text { specimen mass }\end{array}$ & 12.9475 \\
\hline
\end{tabular}

\section{Assembly}

Assembly Drawing

Drawing

S17-42-SIC ENDPLUG

Welding \& Cleaning

X3E020977A633

Rev. $\quad$ Comment

Fill Gas

$46 \% \mathrm{He}$, Ar bal. 


\section{Capsule Number:}

Irradiation Conditions

Irradiation Location

Design Temperature

First Cycle Goal

Irradiation Time

Fill Gas

RRH 7

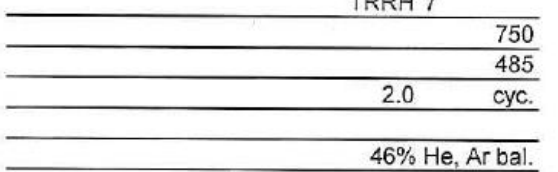

\section{Approvals}

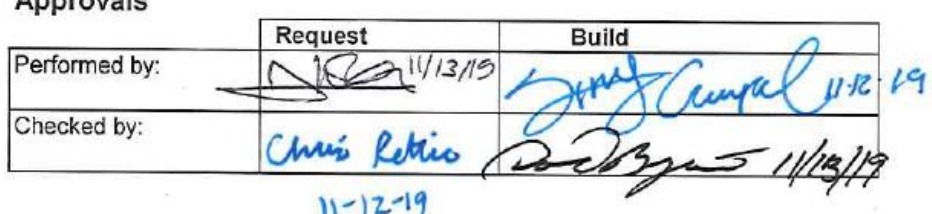

$11-12-19$

Capsule Fabrication

\begin{tabular}{|c|c|c|c|c|c|c|c|c|c|c|}
\hline & Drawing & Rev. & Part & Material & Count & Comment & MAT IR & FAB IR & ID & Mass (g) \\
\hline Housing & X3E020977A634 & C & 1 & Al 6061 & 1 & & 20838 & 20839 & $18-76$ & 4.3127 \\
\hline End Cap & X3E020977A634 & C & 2 & Al 4047 & 1 & & 20823 & 20850 & $18-66$ & 0.6145 \\
\hline Compression Spring & S17-42-SIC_ENDPLUG & 0 & 2 & 304 SS & 1 & & 20659 & 21006 & 09 & 0.1040 \\
\hline Insulator Disk & S17-42-SIC_ENDPLUG & 0 & 3 & Grafoil & 1 & & 19812 & 19812 & 1 total & 0.0086 \\
\hline End Plug Specimen & S17-43-SIC_ENDPLUG & 1 & 1 & $\mathrm{SiC}$ & 1 & HS9 & 20992 & 20992 & HSIC TUBE 9 & 3.8837 \\
\hline Bottom Centering Thimble & S17-43-SIC_ENDPLUG & 1 & 2 & Ti-6Al4V & 1 & $\varnothing 5.64 \pm 0.03$ & 20950 & 20950 & 1 total & 0.2239 \\
\hline Thermometry & S17-43-SIC_ENDPLUG & 1 & 3 & $\mathrm{SiC}$ & 1 & & 19759 & 20948 & HS9 & 3.3789 \\
\hline Top Centering Thimble & S17-43-SIC_ENDPLUG & 1 & 4 & Ti-6Al4V & 1 & & 20950 & 20950 & $18-09$ & 0.2249 \\
\hline & & & & & & & & & $\begin{array}{c}\text { total mass } \\
\text { specimen mass }\end{array}$ & $\frac{12.7512}{3.8837}$ \\
\hline
\end{tabular}

Assembly

\begin{tabular}{|l|c|c|c|}
\cline { 2 - 4 } \multicolumn{1}{c|}{} & \multicolumn{1}{c|}{ Drawing } & Rev. & Comment \\
\hline Assembly Drawing & S17-42-SIC_ENDPLUG & 0 & \\
\hline Welding \& Cleaning & X3E020977A633 & 2 & \\
\hline Fill Gas & \multicolumn{2}{|c|}{$46 \% \mathrm{He}, \mathrm{Ar}$ bal. } \\
\hline
\end{tabular}


Capsule Number:

Irradiation Conditions Irradiation Location

Design Temperature

First Cycle Goa

Irradiation Time

Fill Gas

\section{Capsule Fabrication}

\begin{tabular}{|c|c|c|c|c|}
\hline & & & & \\
\hline & Drawing & Rev. & Part & Material \\
\hline Housing & X3E020977A634 & $\mathrm{C}$ & 1 & Al 6061 \\
\hline End Cap & X3E020977A634 & C & 2 & Al 4047 \\
\hline Compression Spring & S17-42-SIC_ENDPLUG & 0 & 2 & 304 SS \\
\hline Insulator Disk & S17-42-SIC_ENDPLUG & 0 & 3 & Grafoil \\
\hline End Plug Specimen & S17-43-SIC_ENDPLUG & 1 & 1 & $\mathrm{SiC}$ \\
\hline Bottom Centering Thimble & S17-43-SIC_ENDPLUG & 1 & 2 & Ti-6Al4V \\
\hline Thermometry & S17-43-SIC_ENDPLUG & 1 & 3 & $\mathrm{SiC}$ \\
\hline Top Centering Thimble & S17-43-SIC_ENDPLUG & 1 & 4 & $\mathrm{Ti}-6 \mathrm{Al} 4 \mathrm{~V}$ \\
\hline \multirow[t]{2}{*}{ Assembly } & & & & \\
\hline & Drawing & Rev. & \multicolumn{2}{|c|}{ Comment } \\
\hline Assembly Drawing & S17-42-SIC_ENDPLUG & 0 & & \\
\hline Welding \& Cleaning & X3E020977A633 & 2 & \multirow{2}{*}{\multicolumn{2}{|c|}{$46 \% \mathrm{He}$, Ar bal. }} \\
\hline Fill Gas & & & & \\
\hline
\end{tabular}

PGA10

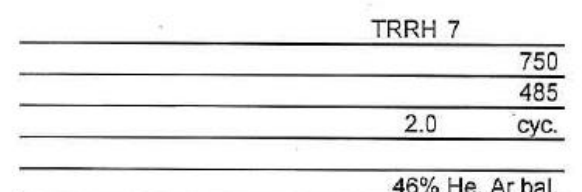

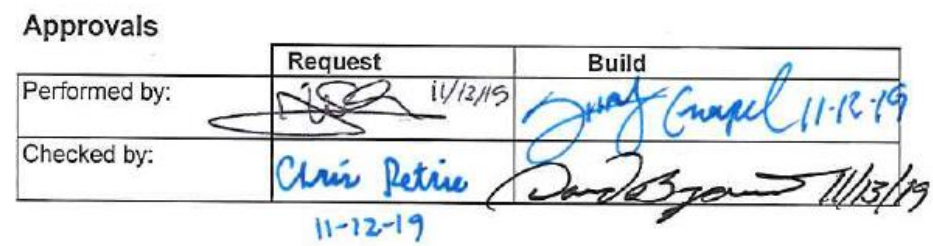

$11-12-19$ 
Capsule Number:

Irradiation Conditions

Irradiation Location

Design Temperature

First Cycle Goal

Irradiation Time

Fill Gas

Capsule Fabrication

\begin{tabular}{|c|c|c|c|c|}
\hline & & & & \\
\hline & Drawing & Rev. & Part & Material \\
\hline Housing & X3E020977A634 & $\mathrm{C}$ & 1 & Al 6061 \\
\hline End Cap & X3E020977A634 & $\mathrm{C}$ & 2 & Al 4047 \\
\hline Compression Spring & S17-42-SIC_ENDPLUG & 0 & 2 & 304 SS \\
\hline Insulator Disk & S17-42-SIC_ENDPLUG & 0 & 3 & Grafoil \\
\hline End Plug Specimen & S17-43-SIC_ENDPLUG & 1 & 1 & $\mathrm{SiC}$ \\
\hline Bottom Centering Thimble & S17-43-SIC_ENDPLUG & 1 & 2 & $\mathrm{Ti}-6 \mathrm{~A} \mid 4 \mathrm{~V}$ \\
\hline Thermometry & S17-43-SIC_ENDPLUG & 1 & 3 & $\mathrm{SiC}$ \\
\hline Top Centering Thimble & S17-43-SIC_ENDPLUG & 1 & 4 & Ti-6Al4V \\
\hline \multicolumn{5}{|l|}{ Assembly } \\
\hline & Drawing & Rev. & \multicolumn{2}{|r|}{ Comment } \\
\hline Assembly Drawing & S17-42-SIC_ENDPLUG & 0 & & \\
\hline Welding \& Cleaning & X3E020977A633 & 2 & \multirow{2}{*}{\multicolumn{2}{|c|}{ Helium }} \\
\hline Fill Gas & & & & \\
\hline
\end{tabular}

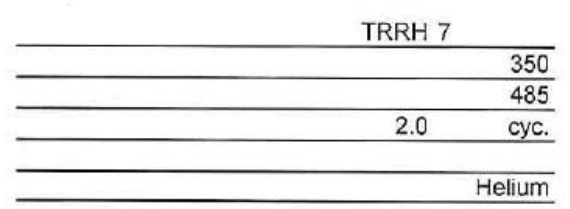

Fill Gas

Helium
Approvals

\begin{tabular}{|c|c|c|c|}
\hline & Request & Build & \\
\hline Performed by: & $1022^{11 / 13 / 19}$ & & \\
\hline Checked by: & Chrie Retio & & $\pi / 134$ \\
\hline
\end{tabular}

II $-12-19$ 


\section{Capsule Fabrication Request Sheet}

\section{Capsule Number:}

Irradiation Conditions

Irradiation Location

\section{First Cycle Goal}

Irradiation Temperature

Housing Fill Gas

\section{Capsule Fabrication}

\begin{tabular}{|c|c|c|c|c|}
\hline & & & & \\
\hline & Drawing & Rev. & Part & Material \\
\hline Housing & X3E0209777A634 & $\mathrm{C}$ & 1 & Al 6061 \\
\hline End Cap & X3E020977A634 & $\mathrm{C}$ & 2 & Al 4047 \\
\hline Center Spacer & S10-05-TTN01 & $\mathrm{C}$ & 2 & Grafoil \\
\hline Retainer Spring & S10-05-TTN01 & $\mathrm{C}$ & 3 & SiC \\
\hline Support Disk & S10-05-TTN01 & $\mathrm{C}$ & 4 & Molybdenum \\
\hline Outer Insulator Disk & S10-05-TTN01 & $\mathrm{C}$ & 5 & Grafoil \\
\hline Insulator Disk & S10-05-TTN01 & C & 6 & Grafoil \\
\hline Specimen Holder Type A & JLM04012008-1 & B & 1 & $\mathrm{~V}-4 \mathrm{Cr} 4 \mathrm{Ti}$ \\
\hline Joint Specimen & S10-05-TTN01 & C & 8 & $\mathrm{SiC}$ \\
\hline Quartz Wool & S10-05-TTN01 & C & 9 & Quartz \\
\hline Centering Thimble & X3E020977A540 & 1 & 1 & Ti-6AI4V \\
\hline Small Thermometry & X3E020977A540 & 1 & 3 & $\mathrm{SiC}$ \\
\hline \multicolumn{5}{|l|}{ Assembly } \\
\hline & Drawing & Rev. & \multicolumn{2}{|l|}{ Comment } \\
\hline Assembly Drawing & S10-05-TTN01 & C & \multirow{2}{*}{\multicolumn{2}{|c|}{ 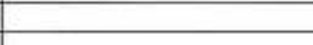 }} \\
\hline Welding \& Cleaning & X3E020977A633 & 2 & & \\
\hline Fili Gas & \multicolumn{2}{|l|}{$\mathrm{Hel}$} & & \\
\hline
\end{tabular}

485

$300^{\circ} \mathrm{C}$
TGA01

$\begin{array}{r}\text { TRRH } \\ \hline \\ \hline 2.0 \text { cycles } \\ \hline 300^{\circ} \mathrm{C} \\ \hline \text { Helium } \\ \hline\end{array}$

Page 1 of 2 Date 11/6/2019

\section{Approvals}

\begin{tabular}{|c|c|}
\hline & Request \\
\hline Performed by: & $72{ }^{116 / 19}$ \\
\hline Checked by: & $\mu_{2}$ Retive ${ }^{11-6-17}$ \\
\hline
\end{tabular}

\section{9}


Capsule Number:

Irradiation Conditions Irradiation Location

First Cycle Goal

Irradiation Time

Irradiation Temperature

Housing Fill Gas

TGA02

$\begin{array}{r}\text { TRRH } \\ \hline \multicolumn{1}{r}{485} \\ \hline 2.0 \text { cycles } \\ \hline 800^{\circ} \mathrm{C} \\ \hline \text { Argon }\end{array}$

\section{Approvals}

\begin{tabular}{|c|c|c|}
\hline \multirow{2}{*}{ Regemed bo } & & \\
\hline & Request & \multirow{2}{*}{ Build } \\
\hline Performed by: & $18022^{11061 / 85}$ & \\
\hline Checked by: & vis betio & \\
\hline
\end{tabular}

Capsule Fabrication

\begin{tabular}{|c|c|c|c|c|c|c|c|c|c|c|}
\hline & Drawing & Rev. & Part & Material & Count & Comment & MAT IR & FAB IR & ID & Mass (g) \\
\hline Housing & X3E0209777A634 & $\mathrm{C}$ & 1 & Al 6061 & 1 & & 20930 & 20930 & $19-24$ & 4.2992 \\
\hline End Cap & X3E0209777A634 & C & 2 & Al 4047 & 1 & & 20823 & 20850 & $18-78$ & 0.6142 \\
\hline Center Spacer & S10-05-TTN01 & $\mathrm{C}$ & 2 & Grafoil & 1 & $6.0 \times 48.0 \times 0.15$ thk & 19812 & 19812 & 1 Total & 0.0373 \\
\hline Retainer Spring & S10-05-TTN01 & C & 3 & $\mathrm{SiC}$ & 2 & & 19759 & 19759 & 2 Total & 0.0554 \\
\hline Support Disk & S10-05-TTN01 & C & 4 & Molybdenum & 2 & $07.5 \times 0.05-0.13$ thk & 19593 & 19593 & 2 Total & 0.06200 \\
\hline Outer Insulator Disk & S10-05-TTN01 & c & 5 & Grafoil & 2 & $09.2 \times 0.05-0.13$ thk & 19812 & 19812 & 2 Total & 0.0178 \\
\hline Insulator Disk & S10-05-TTN01 & C & 6 & Grafoil & 6 & $07.5 \times 0.05-0.13$ thk & 19812 & 19812 & 6 Total & 0.0344 \\
\hline Specimen Holder Type A & JLM04012008-1 & B & 1 & $\mathrm{Nb}-1 \mathrm{Zr}$ & 1 & $9.356 \mathrm{~mm} O D$ & 20339 & 21008 & N2 & 11.2749 \\
\hline \multirow[t]{16}{*}{ Joint Specimen } & \multirow[t]{16}{*}{ S10-05-TTN01 } & \multirow[t]{16}{*}{ c } & \multirow[t]{16}{*}{8} & \multirow[t]{16}{*}{$\mathrm{SiC}$} & \multirow[t]{16}{*}{16} & & \multirow[t]{16}{*}{20989} & \multirow[t]{16}{*}{$20 \$ 89$} & $2 \mathrm{TE}$ & 0.2618 \\
\hline & & & & & & & & & 8TE & 0.2618 \\
\hline & & & & & & & & & $16 \mathrm{GC}$ & 0.2990 \\
\hline & & & & & & & & & $17 \mathrm{GC}$ & 0.2985 \\
\hline & & & & & & & & & $2 \mathrm{HS}$ & 0.2883 \\
\hline & & & & & & & & & $19 \mathrm{HS}$ & 0.3055 \\
\hline & & & & & & & & & $17 \mathrm{HS}$ & 0.3079 \\
\hline & & & & & & & & & $14 \mathrm{HS}$ & 0.3033 \\
\hline & & & & & & & & & 4TE & 0.2617 \\
\hline & & & & & & & & & $11 \mathrm{TE}$ & 0.2621 \\
\hline & & & & & & & & & $4 G C$ & 0.2978 \\
\hline & & & & & & & & & $7 \mathrm{GC}$ & 0.2977 \\
\hline & & & & & & & & & $8 \mathrm{HS}$ & 0.3110 \\
\hline & & & & & & & & & GTE & 0.2633 \\
\hline & & & & & & & & & 10TE & 0.2628 \\
\hline & & & & & & & & & $200 \mathrm{C}$ & 0.2995 \\
\hline Quartz Wool & S10-05-TTN01 & C & 9 & Quartz & AR & & 20679 & 20679 & AR & 0.0201 \\
\hline Centering Thimble & X3E0209977A540 & 1 & 1 & Ti-6Al4V & 2 & & 20536 & 20553 & 2 Total & 0.2796 \\
\hline Small Thermometry & X3E0209977A540 & 1 & 3 & $\mathrm{SiC}$ & 2 & 324,374 & 19502 & 19709 & 2 Tiotal & 0.1324 \\
\hline \multirow{3}{*}{ Assembly } & & & & & & & & & Total Mass & 21.4094 \\
\hline & & & & & & & & & Specimen Mass & 4.7699 \\
\hline & Drawing & Rev. & \multicolumn{2}{|c|}{ Comment } & & & & & Internal Mass & 16.4960 \\
\hline
\end{tabular}

